www.mdpi.com/journal/marinedrugs

Article

\title{
Construction of Escherichia coli Mutant with Decreased Endotoxic Activity by Modifying Lipid A Structure
}

\section{Qiong Liu ${ }^{1}$, Yanyan $\mathrm{Li}^{2}$, Xinxin Zhao ${ }^{1}$, Xue Yang ${ }^{1}$, Qing Liu ${ }^{3, *}$ and Qingke Kong ${ }^{1, *}$}

1 Institute of Preventive Veterinary Medicine, College of Veterinary Medicine, Sichuan Agricultural University, Chengdu 611130, China; E-Mails: p19890528@126.com (Q.L.); xxinzhao@163.com (X.Z.); yx1207@foxmail.com (X.Y.)

2 State Key Laboratory of Food Science and Technology, Jiangnan University, Wuxi 214122, China; E-Mail: yanyanli@jiangnan.edu.cn

3 Department of Bioengineering, College of Veterinary Medicine, Sichuan Agricultural University, Chengdu 611130, China

* Authors to whom correspondence should be addressed; E-Mails: qingke.kong@sicau.edu.cn (Q.K.); qing.liu.2@sicau.edu.cn (Q.L.); Tel.: +86-028-8629-1176.

Academic Editor: Antonio Molinaro

Received: 31 March 2015 / Accepted: 19 May 2015 / Published: 27 May 2015

\begin{abstract}
Escherichia coli BL21 (DE3) and its derivatives are widely used for the production of recombinant proteins, but these purified proteins are always contaminated with lipopolysaccharide (LPS). LPS is recognized by the toll-like receptor 4 and myeloid differentiation factor 2 complex of mammalian immune cells and leads to release of pro-inflammatory cytokines. It is a vital step to remove LPS from the proteins before use for therapeutic purpose. In this study, we constructed BL21 (DE3) $\triangle m s b B 28 \Delta p a g P 38$ mutant, which produces a penta-acylated LPS with reduced endotoxicity. The plasmids harboring $p a g L$ and/or lpxE were then introduced into this mutant to further modify the LPS. The new strain (S004) carrying plasmid pQK004 (pagL and lpxE) produced mono-phosphoryated tetra-acylated lipid A, which induces markedly less production of tumor necrosis factor- $\alpha$ in the RAW264.7 and IL-12 in the THP1, but still retains ability to produce recombinant proteins. This study provides a strategy to decrease endotoxic activity of recombinant proteins purified from $E$. coli BL21 backgrounds and a feasible approach to modify lipid A structure for alternative purposes such as mono-phosphoryl lipid A (MPL) as vaccine adjuvants.
\end{abstract}


Keywords: Escherichia coli BL21 (DE3); lipid A; pagL; lpxE; protein expression

\section{Introduction}

Escherichia coli BL21 (DE3) and its derivatives are widely used for recombinant protein expression due to its high transformation efficiency, large recombinant protein yield and ease of use [1,2]. However, lipopolysaccharide (LPS, also called endotoxin) is co-purified with recombinant proteins from this line of E. coli strains, intrinsically limiting their use in humans and other mammals $[3,4]$. LPS must therefore be removed before BL21-generated recombinant proteins are used for therapeutic purposes or raising antibodies [5]. LPS is a predominant structural component on the cell envelope of Gram-negative bacteria, coating approximately three-fourths of the outer membrane. LPS has three different regions: a hydrophobic lipid A, a short non-repeating core oligosaccharide (C-OS), and a distal polysaccharide termed O-antigen (O-PS) which is the endmost distal to the outer membrane [1,6,7]. Lipid A is the component of LPS that can activate both the TLR4/MD-2/CD14 pathway and caspase 11 pathway [8] resulting in an immuno-inflammatory response. Overstimulation of innate immunity by lipid A will lead to organ damage, severe shock, even death of mammals and humans $[9,10]$. As lipid A is a minimum structural component necessary for $E$. coli BL21 growth in the laboratory condition [11], we cannot remove lipid A structure from E. coli BL21 to avoid LPS contamination during protein purification; however, we are able to modify lipid A structure to decrease or eliminate its endotoxic property by genetic engineering approaches [8].

E. coli lipid A contains two phosphate groups and six acyl chains, which is the most efficient form for activating pro-inflammatory responses [6]. Several proteins are involved in altering the number of acyl chains in E. coli lipid A, including HtrB (LpxL), MsbB (LpxM), PagP, PagL and LpxR. LpxL and LpxM are responsible for the addition of acyl chains in lipid A synthesis, and PagP controls the late decoration of acyl chains. LpxR and PagL will catalyze removal of an acyl chain from lipid A [12]. $m s b B$ encodes a ligase responsible for addition of the myristic acid moiety to the lipid A in the last step of lipid A synthesis, though not essential for bacterial growth [13], as the $\triangle m s b B$ mutant grows normally and produces a non-myristylated LPS [12]. The lipid A from E. coli msbB mutant lacking myristoyl fatty acid moiety could reduce the ability to stimulate immune cells to produce E-selectin and TNF- $\alpha$ [14-16]; for example, $\Delta m s b B$ derivatives have been used to develop live vaccines to treat cancer [17]. PagP catalyzes addition of a phospholipid-derived palmitate chain to the hydroxyl of the $R$-3'-hydroxymyristate chain at the 2 position of lipid A $[18,19]$. A pagP gene is present in the genome of $E$. coli BL21 (DE3), which is not active when grown under normal conditions. Palmitoylation of lipid A by PagP will lead to an increased resistance to cationic antimicrobial peptides (CAMPs) in E. coli [19-21]. Although it was not discovered in E. coli, PagL derived from S. typhimurium was shown to hydrolyze the ester bond at the position of lipid A and also was able to function in E. coli, thereby releasing the primary 3-hydroxymyristoyl moiety [22,23]. Deacylation by PagL modulates the recognition of lipid A by the TLR4/MD-2 complex and decreases its ability to induce pro-inflammatory response [24,25]. LpxE, an inner membrane phosphatase from Francisella tularensis subspecies novicida can selectively remove the 1'-phosphate group of lipid A in E. coli and 
Salmonella [26,27], generating a structure similar to mono-phosphoryl lipid A (MPL) that remains covalently linked to LPS.

The total number, length and position of the acyl chains and two phosphate groups at the 1 and 4' positions are critical factors for full lipid A activation of TLR4/MD2 and caspase-11 pathway [28-31]. One strategy to decrease the endotoxic activity is to reduce the number of fatty acyl chain and/or remove phosphate group from lipid A structure [32]. In this study, we aim at maximally reducing lipid A endotoxicity by altering the number of fatty acid chains and removing 1'-phosphate group of lipid A via deleting $m s b B$ and $p a g P$ and overexpressing $l p x E$ and $p a g L$ in the BL21 (DE3) strain for the purpose of expressing proteins with low endotoxic activity.

\section{Materials and Methods}

\subsection{Bacterial Strains, Plasmids, Media, and Growth Conditions}

Escherichia coli BL21 (DE3) (NEB) and its derivatives were routinely grown at $37{ }^{\circ} \mathrm{C}$ in lysogeny broth (LB) [33] or on LB agar. When required, antibiotics were added at an appropriate final concentration $(\mu \mathrm{g} / \mathrm{mL}), 50$ for kanamycin $(\mathrm{Kan}), 25$ for chloramphenicol $(\mathrm{Cm})$. Diaminopimelic acid (DAP) was added to a concentration of $50 \mu \mathrm{g} / \mathrm{mL}$ for the growth of E. coli strain $\chi 7213$ [34]. LB agar containing $5 \%$ sucrose was used for $s a c B$ gene-based counter-selection in allelic-exchange experiments.

\subsection{Construction of Plasmids and Bacterial Strains}

DNA manipulations were carried out as described [35]. Transformation of E. coli was performed by electroporation. Transformants were selected on LB agar plates containing appropriate antibiotics. The primers used in this study are listed in Table 1 . For construction of the $\triangle m s b B$ mutation, which deleted the entire $m s b B$ open reading frame, the BL21 (DE3) genome was used as a template for PCR. Fragments in the amount of 250-bp DNA containing the region upstream of the $m s b B$ gene and downstream of the $m s b B$ gene using primers $m s b B-1 F / 1 R$ and $m s b B-2 F / 2 R$ were amplified, respectively (Table 1). The two PCR fragments were purified in agarose gels, combined at a 1:1 molar ratio, and joined by PCR using primers msbB- $1 \mathrm{~F}$ and $\mathrm{msbB}-2 \mathrm{R}$. The resulting PCR product with A-terminal was inserted to T-terminal pRE112 vector generated by AhdI digestion [36], resulting in plasmid pQK001. The same strategy was used to construct pQK002 for pagP deletion in BL21 (DE3) (Table 2).

The plasmid pYA4291 containing a fragment of $\Delta l p x R: \mathrm{P}_{\mathrm{pp}}$ pagL was used as a template for constructing a plasmid to express pagL. Briefly, the fragment of lpxRU-Plpp pagL-lpxRD was amplified using primers lpxRscreenF and lpxRscreenR, and ligated to a T-terminal vector $p 15 \mathrm{a}$ generated by $A h d \mathrm{I}$ digestion, resulting in plasmid pQK003. Using the same strategy, pQK004 was constructed to express $l p x E$ based on pYA4295 [37]. pQK005 was constructed by inserting the fragment of lpxRU-Plpp pagL-lpxRD to blunt plasmid pQK004 generated by SnaBI digestion (Table 2).

A plasmid pQK006 was constructed for protein expression in BL21. A phoP gene was amplified from the genome of Pasteurella multocida using the primer pairs phoP-F/phoP-R which contained restriction sites NdeI and BamHI. The PCR product was digested and ligated to the same enzyme-digested 
vector $\mathrm{pET}-32 \mathrm{a}$, resulting in $\mathrm{pQK} 005$. All the plasmids in this study were sequenced to confirm their correct insertion.

Table 1. Primers used in this study.

\begin{tabular}{|c|c|c|}
\hline Primers & Sequences $\left(5^{\prime}-3^{\prime}\right)$ & Function \\
\hline $\mathrm{msbB}-1 \mathrm{~F}$ & CAGTTCGACAATGTGGAAGAAG & \multirow{4}{*}{$\begin{array}{c}\text { For deletion of } m s b B \text { by } \\
\text { suicide plasmid }\end{array}$} \\
\hline $\mathrm{msbB}-1 \mathrm{R}$ & ACCTGCAGGATGCGGCCGCGG GCCTCTCGCGAGG & \\
\hline $\mathrm{msbB}-2 \mathrm{~F}$ & CCGCGGCCGCATCCTGCAGGT GCTTTTCCAGTTTCGG & \\
\hline $\mathrm{msbB}-2 \mathrm{R}$ & GCGTTATATGCACTTGCGC & \\
\hline pagP-1F & CCTTGATTGCATTTTGTCAT & \multirow{4}{*}{$\begin{array}{l}\text { For deletion of pagP by } \\
\text { suicide plasmid }\end{array}$} \\
\hline pagP-1R & GTCTCACCCGGGCCTGCAGGTTGTGACCATAAAACATTTA & \\
\hline pagP-2F & CACAACCTGCAGGCCCGGGTGAGACAAATGAAGTTTTAGT & \\
\hline pagP-2R & TGCTGCCGTCTTCCGGAGTA & \\
\hline pagPscreenF & AAACGCCGTTAACCCGATA & \multirow{2}{*}{ Insert $\mathrm{P}_{\mathrm{lpp}} l p x E$ to $\mathrm{p} 15 \mathrm{a}$ vector } \\
\hline pagPscreenR & TAGACACAAATGCTGCTGTGTCG & \\
\hline lpxRscreenF & CAGGGGGTGTCAGTATTTGGCG & \multirow{2}{*}{ Insert $\mathrm{P}_{\mathrm{lpp}}$ pagL to $\mathrm{p} 15 \mathrm{a}$ vector } \\
\hline lpxRscreenR & CGTGAAGCCAATAATTTCTCGCAC & \\
\hline phoP-F & TCATATGCGAATTTTATTAATAGAATATG & \multirow{2}{*}{$\begin{array}{l}\text { Insert } p h o P \text { gene to } \mathrm{pET}-32 \mathrm{a} \\
\text { for expression phoP protein } \\
\text { from Pasteurella }\end{array}$} \\
\hline phoP-R & TGGATCCTTAAGCCATTTCATCATTTTTTCC & \\
\hline
\end{tabular}

Table 2. Bacterial strains and plasmids used in this study.

\begin{tabular}{|c|c|c|}
\hline Strains or Plasmids & Description & Source \\
\hline \multicolumn{3}{|c|}{ Strains } \\
\hline E. coli BL21 (DE3) & Expression protein strain & NEB \\
\hline $\mathrm{S} 001$ & BL21 (DE3) $\Delta m s b B 28 \triangle p a g P 38$ & This work \\
\hline S002 & S001 with pQK003 & This work \\
\hline S003 & S001 with pQK004 & This work \\
\hline S004 & S001 with pQK005 & This work \\
\hline$\chi 7232$ & 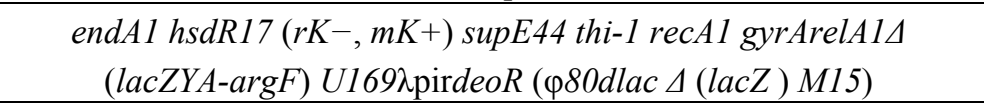 & [37] \\
\hline$\chi 7213$ & $\begin{array}{l}\text { thi-1 thr-1 leuB6 glnV44 tonA21 lacY1 recA1 } \\
\text { RP4-2-Tc: } \mu \lambda \text { pir } \Delta a s d A 4 \Delta z h f-2: T n 10\end{array}$ & [37] \\
\hline \multicolumn{3}{|c|}{ Plasmids } \\
\hline p15a T-vector & Low copy expression plasmid & {$[38]$} \\
\hline pET-32a & Protein expression & Novagen \\
\hline pQK001 & For deletion of $m s b B$ in BL21 & This work \\
\hline pQK002 & For deletion of pagP in BL21 & This work \\
\hline pYA4291 & $\mathrm{P}_{\mathrm{lpp}}$ pagL in pRE112 & Lab collection \\
\hline pYA4295 & $\mathrm{P}_{\mathrm{lpp}} \operatorname{lpxE}$ (codon-optimized) in pRE112 & [39] \\
\hline pQK003 & Insert $\mathrm{P}_{\mathrm{lpp}}$ pagL to $\mathrm{p} 15 \mathrm{a}$ and express PagL for deacylation of lipid $\mathrm{A}$ & This work \\
\hline pQK004 & $\begin{array}{c}\text { Insert } \mathrm{P}_{\mathrm{lpp}} l p x E \text { to } \mathrm{p} 15 \mathrm{a} \text { and express } l p x E \text { to remove the } \\
\text { 1-phosphate group of lipid } \mathrm{A}\end{array}$ & This work \\
\hline pQK005 & $\begin{array}{l}\text { Insert } \mathrm{P}_{\mathrm{lpp}} \text { pagL and } \mathrm{P}_{\mathrm{lpp}} l p x E \text { to p15a Express both } p a g L \text { and } l p x E \text { to } \\
\text { modify the lipid A }\end{array}$ & This work \\
\hline pQK006 & Expression of PhoP protein of Pasteurella multocida & Lab collection \\
\hline
\end{tabular}




\subsection{BL21 (DE3) Mutant Strain Construction}

$\triangle m s b B$ and $\triangle p a g P$ mutation in the BL21 (DE3) were constructed by suicide plasmids method [40]. Briefly, the bacterial cultures of the BL21 (DE3) and the E. coli $\chi 7213$ harboring suicide plasmids pQK001 (for $\triangle m s b B$ ) or pQK002 (for $\triangle p a g P$ ) were conjugated at the ratio of 1:2 on LB solid agar plate overnight, and then the mixed bacteria were streaked on LB plates containing $25 \mu \mathrm{g} / \mathrm{mL} \mathrm{Cm}$ and incubated overnight. Isolated colonies, in which the suicide plasmid was integrated into the BL21 genome, were grown on LB Cm plates. Cm-resistant colonies were propagated in LB liquid media $4 \mathrm{~h}$, diluted, and spread on LB plates containing 5\% sucrose. Colonies which have a sucrose-resistant and $\mathrm{Cm}$-sensitive phenotype were screened by colony PCR. Using the same strategy, the second mutation $\triangle p a g P$ could be introduced into the $\triangle m s b B$ mutant to generate double mutant S001.

\subsection{Analysis of Characteristics of BL21 (DE3) Mutant Strains}

Ten microliters of BL21 (DE3) and mutant strains were inoculated into a tube containing $2 \mathrm{~mL}$ of LB liquid medium and incubated overnight at $37^{\circ} \mathrm{C}$. Of these cultures, $1 \mathrm{~mL}$ was subsequently grown in $99 \mathrm{~mL} \mathrm{LB}$ liquid medium and growth was estimated at $1 \mathrm{~h}$ intervals by measuring the optical density $\left(\mathrm{OD}_{600}\right)$ of the culture using the Spectronic 20. Growth curves were measured in triplicate using three biological replicates.

Plasmid pQK006 was introduced into competent BL21 (DE3) and modified progeny strains, and transformants were grown to an $\mathrm{OD}_{600}$ of 0.6 at $37{ }^{\circ} \mathrm{C}$ in $\mathrm{LB}$ medium supplemented with $50 \mu \mathrm{g} / \mathrm{mL}$ kanamycin and $25 \mu \mathrm{g} / \mathrm{mL} \mathrm{Cm}$ when needed. Expression of recombinant protein was induced by addition of isopropyl $\beta$-D-1-thiogalactopyranoside (IPTG) (final concentration, $1 \mathrm{mM}$ ) for $4 \mathrm{~h}$. Bacteria were harvested and lysed by sonication in $25 \mathrm{mM}$ Tris-HCl $\mathrm{pH} 8.0,1.15 \mathrm{mM}$ EDTA, $1 \mathrm{mg} / \mathrm{mL}$ lysozyme. Cell lysates were centrifuged at $12,000 \times g$ for $5 \mathrm{~min}$. The supernatant contained the soluble fraction while the pellets were the insoluble material. Fractions were suspended in SDS-PAGE loading buffer (50 mM Tris- $\mathrm{HCl} \mathrm{pH}$ 6.8, $0.1 \mathrm{M}$ dithiothreitol, 2\% SDS, $0.1 \%$ bromophenol blue, $10 \%$ glycerol). The different fractions were separated by SDS-PAGE and stained by Coomassie blue.

\subsection{Lipid A Isolation and Mass Spectrometry Procedures}

Lipid A isolation was performed as described previously with some modifications [27]. Briefly, bacteria in $200 \mathrm{~mL}$ cultures were grown to an $\mathrm{OD}_{600}$ of 1.0. The cells were pelleted and washed with PBS twice. The cell pellet was resuspended in a total volume of $76 \mathrm{~mL}$ single-phase Bligh-Dyer mixture containing chloroform, methanol and water $(1: 2: 0.8 \mathrm{v} / \mathrm{v})$, mixed and agitated for $1 \mathrm{~h}$ at room temperature. The cell fragments were harvested by centrifugation at $2000 \mathrm{rpm}$ for $20 \mathrm{~min}$, and the pellets were washed twice using the same solution to remove the soluble material. A total of $25 \mathrm{~mL}$ $12.5 \mathrm{mM}$ sodium acetate $(\mathrm{pH} 4.5)$ was added to suspend the pellets, which were sonicated and then heated to $100{ }^{\circ} \mathrm{C}$ for $30 \mathrm{~min}$ for releasing lipid A from LPS. After cooling to room temperature, the suspensions were re-extracted by two-phase Bligh-Dyer mixtures containing chloroform, methanol and water $(2: 2: 1.8, \mathrm{v} / \mathrm{v} / \mathrm{v})$. The samples were vortexed to extract lipid A and then centrifuged at $2000 \mathrm{rpm}$ for 20 min to separate. The lower phase containing lipid A was transferred to a round-bottom flask and 
dried through a rotary evaporator. The dried lipid A samples were solubilized in chloroform/methanol $(2: 1, \mathrm{v} / \mathrm{v})$ and stored at $-80^{\circ} \mathrm{C}$.

Negative ion Matrix Assisted Laser Desorption Ionization (MALDI)-TOF MS experiments were performed as described elsewhere [41]. Briefly, lipids were solubilized in $100 \mu \mathrm{L}$ chloroform/methanol $(2: 1, \mathrm{v} / \mathrm{v})$ and spotted $(1 \mu \mathrm{L})$ directly onto the MALDI sample plate, followed by $1 \mu \mathrm{L}$ of $100 \mathrm{mg} / \mathrm{mL}$ norharmane MALDI matrix dissolved in chloroform/methanol/water (3:1.5:0.25, v/v/v). All experiments were performed using a Bruker Autoflex Speed MALDI-TOF/TOF mass spectrometer (Bruker Daltonics Inc., Billerica, MA, USA). Each spectrum was an average of 500 shots and 50\% laser power. ES Tuning Mix (Agilent, Palo Alto, CA, USA) was used as a calibration standard. Norharmane matrix was used throughout these studies.

\subsection{Purification and Concentration Measurement of Lipopolysaccharide (LPS) from BL21 (DE3) and Its Mutants}

LPS were purified from BL21 (DE3) and mutant strains by the phenol-water procedure with some modification [42]. Harvested bacteria (500 mg) were suspended with $15 \mathrm{~mL}$ of $10 \mathrm{mM}$ Tris-Cl buffer (pH 8.0), containing 2\% SDS, $4 \%$ 2-mercaptoethanol, and $2 \mathrm{mM} \mathrm{MgCl}_{2}$ in a centrifuge vessel. The suspension was placed in a $65{ }^{\circ} \mathrm{C}$ water bath until bacteria were solubilized. A total of $100 \mu \mathrm{g}$ proteinase $\mathrm{K}$ was added in the suspension and the sample was incubated at $65{ }^{\circ} \mathrm{C}$ water bath for an hour, after which the sample was placed in a $37{ }^{\circ} \mathrm{C}$ water bath overnight. Two $\mathrm{mL}$ of $3 \mathrm{M}$ sodium acetate was added to the proteinase K-digested cell suspension and then $40 \mathrm{~mL}$ of pre-chilled ethanol was added to the bacteria suspension and incubated at $-20^{\circ} \mathrm{C}$ overnight to form precipitate. The sample was centrifuged at $4000 \times g$ for $15 \mathrm{~min}$ and supernatant was decanted slowly; the precipitate was resuspended in $9 \mathrm{~mL}$ of distilled water. One $\mathrm{mL}$ of $3 \mathrm{M}$ sodium acetate was added in the mixture before adding another $20 \mathrm{~mL}$ of chilled absolute ethanol, vortexing after each step. The suspension was placed at $-20{ }^{\circ} \mathrm{C}$ overnight. After centrifugation, the precipitate was suspended by $9 \mathrm{~mL} 10 \mathrm{mM}$ Tris-Cl (pH7.4), and then, the mixture sample was placed at $37^{\circ} \mathrm{C}$ for $4 \mathrm{~h}$ with $50 \mu \mathrm{g}$ DNase I and $12.5 \mu \mathrm{g}$ RNase to digest residual contaminating nucleic acids. To remove all residual protein contaminants from LPS nuclease-treated mixture, the next step was a phenol extraction. The LPS mixture was placed in a $65{ }^{\circ} \mathrm{C}$ water bath for $30 \mathrm{~min}$ and an equal volume of $90 \%$ phenol that preheated to $65^{\circ} \mathrm{C}$ was added. Subsequently, the mixture was placed at $65{ }^{\circ} \mathrm{C}$ for $15 \mathrm{~min}$, cooled to $4{ }^{\circ} \mathrm{C}$ on ice and then centrifuged at $6000 \times \mathrm{g}$ for $15 \mathrm{~min}$. The aqueous top layer was collected in a new vessel and the phenol layer extracted with an equal volume of distilled water again. The mixture sample was heated to $65{ }^{\circ} \mathrm{C}$ again for $15 \mathrm{~min}$ and then placed in an ice-water environment to chill. After centrifugation, the top aqueous layer was added to the first aqueous extraction, all aqueous layers were dialyzed against multiple changes of distilled water over 2 days. After dialysis, the lipopolysaccharide was lyophilized and then dissolved in water to be stored at $-80{ }^{\circ} \mathrm{C}$ until analyzed.

As the molecular weight of LPS samples from BL21 (DE3) and its derivatives are variable, and LPS molecule interacts with MD2-TLR4 to form a complex with a molecular ratio (1:1:1) to initiate the intracellular signaling, LPS samples were quantitated using the 3-deoxy-D-manno-octulosonic acid (Kdo) method according to published procedures [43], and the LPS were calculated and diluted to the 
appropriate concentration (Molar) for each experiment based on two Kdo moieties present in the LPS of the BL21 (DE3) and its derivatives.

\subsection{LPS Stimulation of the Murine Macrophage Cell RAW264.7 and Human Monocyte Cell THP1}

Murine macrophage cell line RAW264.7 and human monocyte cell THP1 were obtained from the cell bank of the Chinese Academy of Science (Shanghai, China). The cell lines were maintained at $37{ }^{\circ} \mathrm{C}$ with 5\% $\mathrm{CO}_{2}$ in DMEM (Gibco BRL, Gaithersburg, MD, USA) containing 10\% FBS (Hyclone, Logan, UT, USA) and in RPMI-1640 Medium (Gibco BRL, Gaithersburg, MD, USA) containing 10\% FBS (Hyclone, Logan, UT, USA), respectively. For cell differentiation, THP-1 cells were treated with $10 \mathrm{mM}$ phorbol 12-myristate 13-acetate (PMA) (Sigma-Aldrich, St. Louis, MO, USA) and incubated at $37{ }^{\circ} \mathrm{C}$ in $5 \% \mathrm{CO}_{2}$ for $48 \mathrm{~h}$. Cells were cultured to the concentration of $10^{5}$ per milliliter and seeded into 96-well plates with $200 \mu \mathrm{L}$ medium each well. After $18 \mathrm{~h}$, cells were stimulated with increasing concentration of LPS $\left(10,10^{2}, 10^{3}\right.$ and $\left.10^{4} \mathrm{pM}\right)$. After $24 \mathrm{~h}$, culture supernatants were collected, centrifuged to pellet debris and stored at $-80{ }^{\circ} \mathrm{C}$ for cytokine analysis. The concentrations of TNF- $\alpha$ and IL-12 were determined using the enzyme-linked immunosorbent assay kit (R \& D Systems, Minneapolis, MN, USA), according to the manufacturer's instruction.

One-way analysis of variance (ANOVA) was performed to determine the statistical significance of the differences between mean values for various experimental and control groups. Data were expressed as means \pm one standard deviation, and the experiments were performed with three biological replicates. The means were compared using the least significant difference test. $p<0.05$ was considered a significant difference and $p<0.01$ was considered extremely significant difference. All data were analyzed with Statistical Product and Service Solutions (SPSS) Statistics 17.0 (Chicago, IL, USA).

\section{Results}

\subsection{Construction of BL21 (DE3) $\triangle m s b B 28 \Delta$ pagP38 Mutant and Its Derivatives}

Two suicide plasmids pQK001 and pQK002 were constructed to facilitate deletion of $m s b B$ and pagP gene in the BL21 (DE3), respectively, and the gene deletions were performed by homologous recombination via SacB-based counter-selection in allelic-exchange experiments [37]. Figure 1 illustrates the chromosomal structures of $\triangle m s b B 28$ and $\triangle p a g P 38$ in BL21 (DE3), and their PCR product size when amplified by flanking primers. The intermediate single $\triangle m s b B 28$ or $\triangle p a g P 38$ mutants were further confirmed by DNA sequencing. $\triangle p a g P 38$ was introduced into intermediate mutant $\triangle m s b B 28$ to yield the mutant S001 ( $\triangle m s b B 28 \Delta p a g P 38)$, which was hypothesized to result in production of penta-acylated lipid A in this progeny mutant strain (Table 2).

The other three plasmids were constructed for overexpressing pagL (pQK003), lpxE (pQK004), and both pagI and IpxE (pQK005) under constitutive transcriptional control of the strong E. coli promoter $\mathrm{P}_{\text {lpp }}$ (Figure 2). These plasmids replicate with low copy number in E. coli (origin of replication p15a) and encode a $\mathrm{Cm}$ resistance marker to facilitate use in other bacterial strains. Plasmids pQK003, pQK004 and pQK005 were transformed separately to S001 to yield the strains S002, S003 and S004, respectively (Table 2 ). 


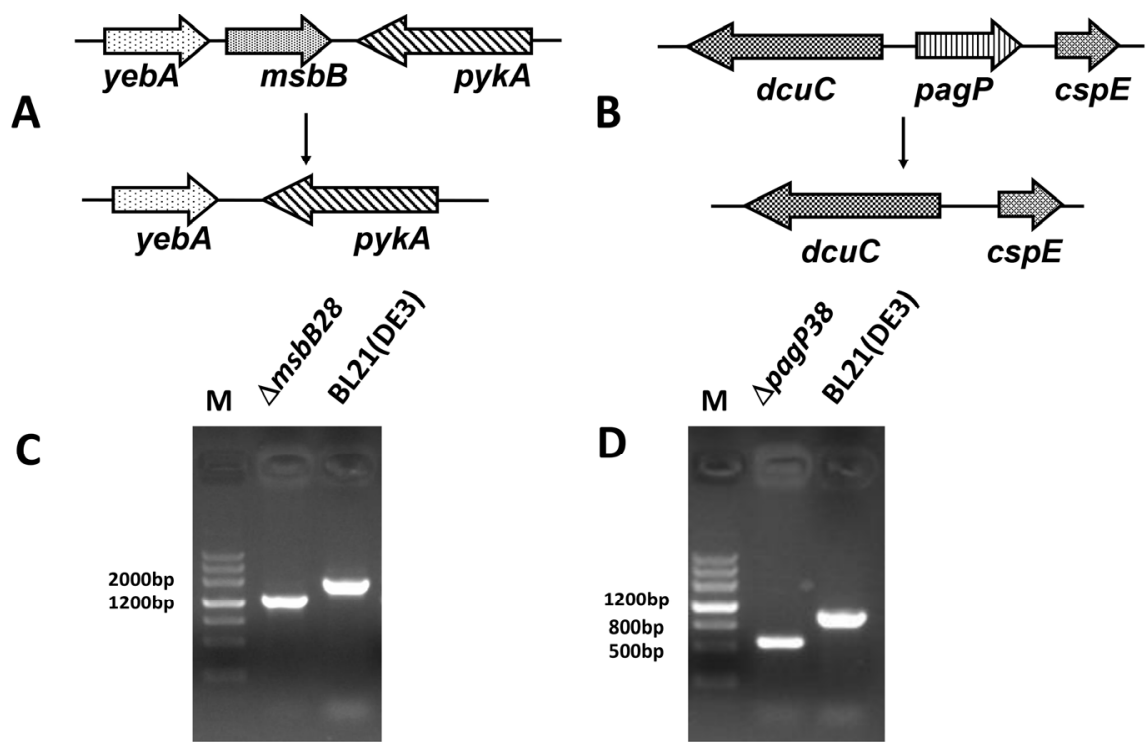

Figure 1. Construction of BL21 (DE3) mutant strain. (A) Map of deletion mutant resulting from knockout of $m s b B$ gene; (B) Map of deletion mutant resulting in knockout of pagP gene; (C) The identification result of $\triangle m s b B 28$ mutant, the result shows that $m s b B$ gene was deleted; (D) The identification result of $\triangle p a g P 38$ mutant, the result shows that pagP gene was deleted.
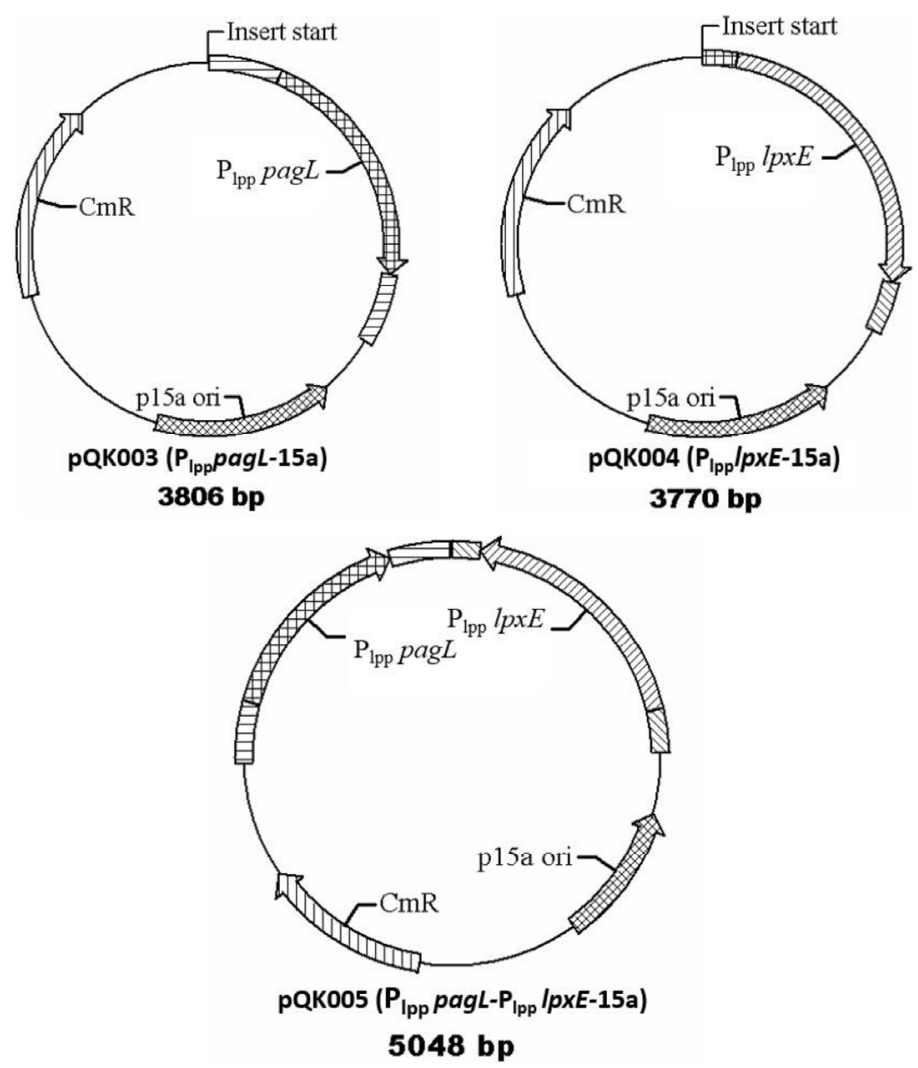

Figure 2. Plasmid maps used in this study. Maps of three expression plasmids constructed in this study. Plasmid pQK003 was constructed for expressing pagL to decrease the fatty acyl chain number. Plasmid pQK004 was constructed for expressing lpxE to remove 1-phosphate group in lipid A. Plasmid pQK005 was constructed for expressing pagL and lpxE to confer both of these functions. 


\subsection{Structure Analysis of Lipid A Extracted From BL21(DE3) and Its Derivatives}

Lipid A were extracted from BL21(DE3) and its derivatives by the Bligh-Dyer method [44], and analyzed by MALDI-TOF-MS (Figures 3 and 4). The lipid A isolated from parent strain BL21(DE3) contains a predominant hexa-acylated lipid A ( $\mathrm{m} / \mathrm{z} 1796.2$ in Figure 4) and a minor peak of hexa-acylated lipid A decorated with a 4-amino-4-deoxy-L-arabinose (L-Ara4N) residue $(\mathrm{m} / \mathrm{z} 1950.1)$, which is consistent with other reports [45]. S001 ( $\triangle m s b B 28 \Delta p a g P 38)$ yielded a major peak at $m / z 1586.3$ in the spectrum, resulting from failure of myristic acid chain addition to the 3 '-position of lipid $A$ due to the $m s b B$ deletion (Figures 3 and 4B), and there was the second major peak at $m / z 1739.5$, which was a penta-acylated lipid A modified with L-Ara4N. As expected, PagL expression in the strain S002 lead to a major peak of 3-O-deacylated lipid A at $\mathrm{m} / \mathrm{z} 1359.8$ in the spectrum, which is tetra-acylated lipid A (Figures 3 and 4C), and LpxE expression resulted in production of mono-phosphorylpenta-acylated lipid A ( $\mathrm{m} / \mathrm{z} 1506.1)$ and a minor peak at $\mathrm{m} / \mathrm{z} 1629.1$ which is a mono-phosphorylpenta-acylated lipid A derivative of a phosphoethanolamine ( $\mathrm{pEtN}$ ) addition to the 4'-phosphate position in the strain S003 (Figure 4D); in the spectrum of lipid A isolated from the strain S004 with both PagL and LpxE expression, there are two major peaks at $\mathrm{m} / \mathrm{z} 1279.9$ and $\mathrm{m} / \mathrm{z} 1402.9$ and a minor peak at $\mathrm{m} / \mathrm{z} 1506.1$; the peak at $m / z 1279.9$ is a dephosphorylated tetra-acylated lipid $\mathrm{A}$ at the 1-position, and a pEtN addition to dephosphorylated tetra-acylated lipid A at the 4'-position lead to the peak at $\mathrm{m} / z$ 1402.9, and the peak at $\mathrm{m} / z$ 1506.1 is a mono-phosphorylpenta-acylated lipid A, indicating that PagL is not fully functional in the strain S004.

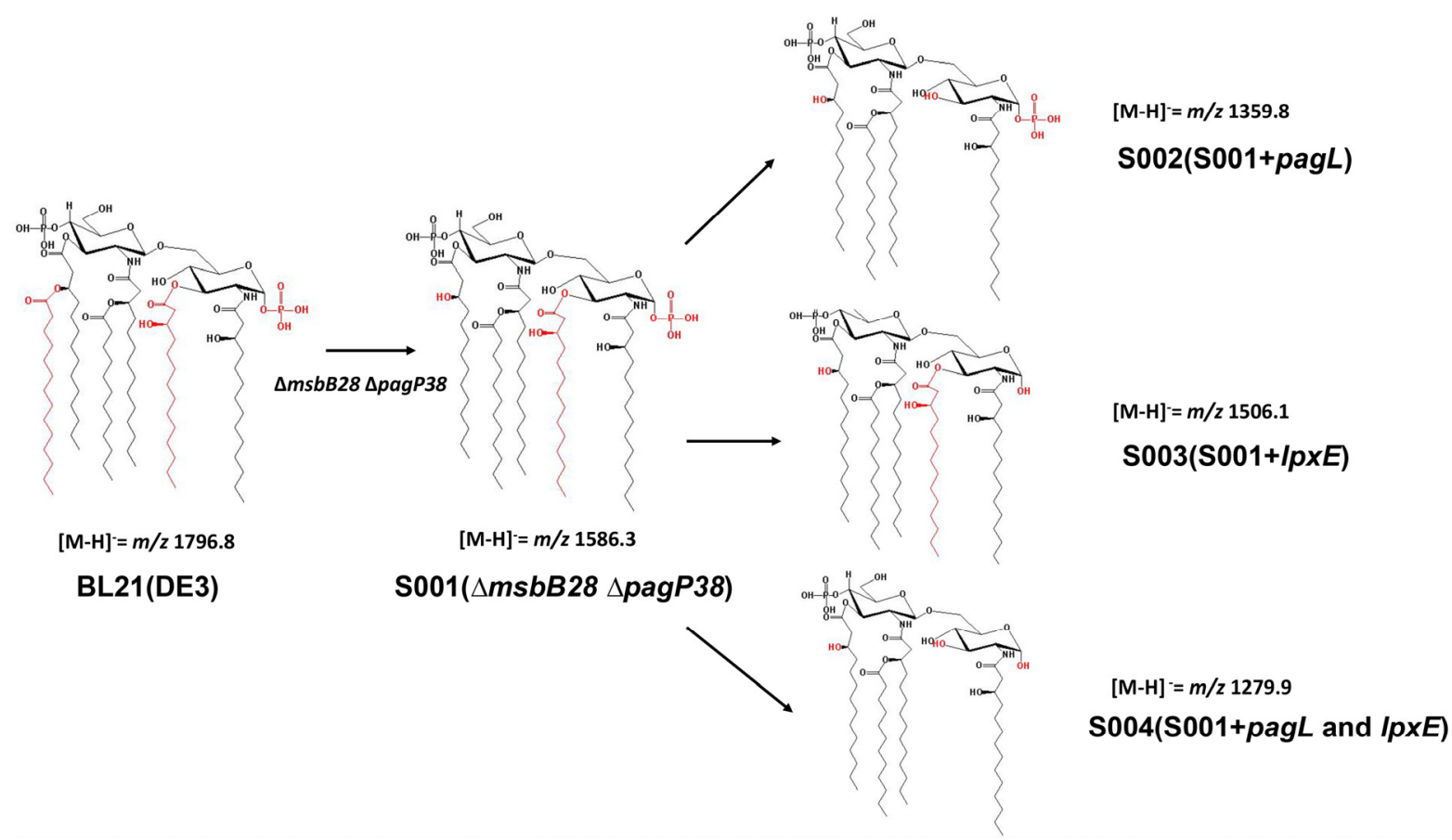

Figure 3. The schematic structure of lipid A. The number of phosphate and fatty acyl chain of lipid A is indicated and the spectrum number $m / z$ of lipid A structure is shown. The deletion of $m s b B$ and overexpression of pagL could change the number of fatty acyl chains of lipid A. Expression of $\operatorname{lp} x E$ in $E$. coli could remove the phosphate group of lipid A. Both structural modifications therefore change lipid A to tetra-acylated monophosphoryl lipid A. 


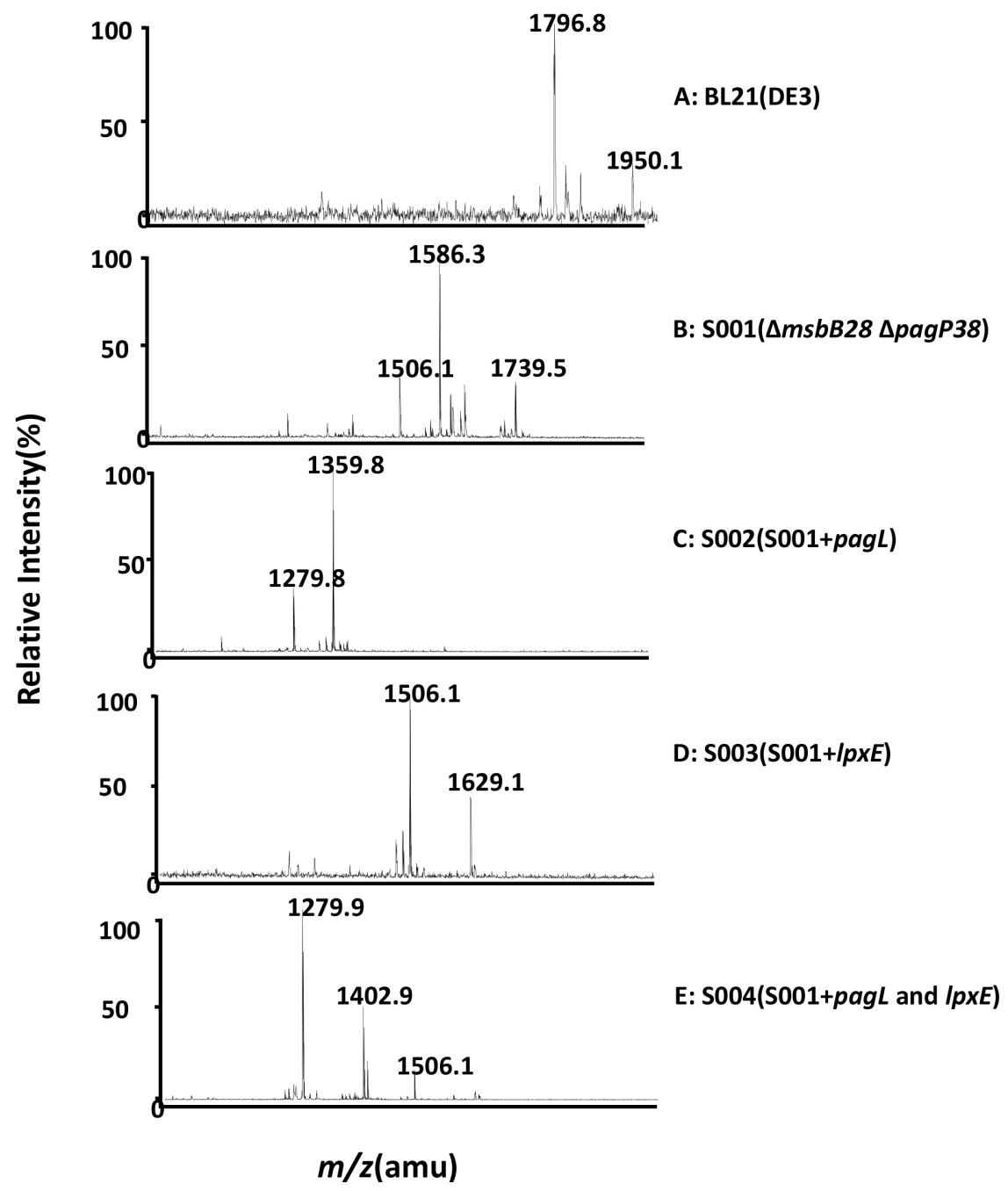

Figure 4. MALDI-TOF MS analysis of lipid A isolated from BL21 (DE3) mutants. Lipid A samples were dissolved in chloroform/methanol (4:1, v/v) and subjected to MALDI-TOF MS in the negative ion mode. The parental strain BL21 (DE3) makes a hexa-acylated lipid A as indicated by the $[\mathrm{M}-\mathrm{H}]^{-}$peak at $m / z$ 1796.8. The mutant strain S001 makes a penta-acylated lipid A as indicated by the $[\mathrm{M}-\mathrm{H}]^{-}$peak at $m / z 1586.3$. Removal of one phosphate group shifts the lipid A $[\mathrm{M}-\mathrm{H}]^{-}$peak by $m / z-70$. Removal of a fatty aycl chain shifts the MS peak by $m / z-226.5$.

There are some other minor peaks in the lipid A mass spectrum isolated from the BL21(DE3) strain and its derivatives, such as peak at $\mathrm{m} / \mathrm{z} 1506.1$ in the S001 and peak at 1279.8 in strain S002, which might arise by loss of a phosphate due to the harsh hydrolysis of cleavage of the lipid A from the core-saccharide, or may result from the small molecules addition to the lipid A when performing lipid A analysis by mass spectrum [27].

\subsection{Comparison of the Endotoxic Activities of LPS Isolated from BL21 (DE3) and Its Derivatives}

The effect of modified lipid A on cytokine stimulation in tissue culture was determined by measuring TNF- $\alpha$ in macrophage cell line RAW264.7 originated from mouse and THP1 cells derived from human, representing two distinct TLR4 receptors [46,47]. LPS was purified from BL21 (DE3) 
and its derivatives by hot-phenol method and repurified with deoxycholate-phenol to eliminate lipoproteins, ensuring no other known pathogen-associated molecular patterns (PAMPs) were present in the LPS samples when measuring endotoxic activity [48].

The TNF- $\alpha$ in the RAW264.7 and IL-12 in the THP1 were measured after $24 \mathrm{~h}$ stimulation by LPS of various concentrations from $10 \mathrm{pmol} / \mathrm{L}$ to $10^{4} \mathrm{pmol} / \mathrm{L}$ (Figure 5 and Supplementary Table S1). The levels of TNF- $\alpha$ in the RAW264.7 were proportional to the concentration of LPS isolated from BL21 (DE3) and its derivatives, and all types of LPS including tetra-acylated and dephosphorylated lipid A acts as agonists to activate the mouse TLR4-pathway.

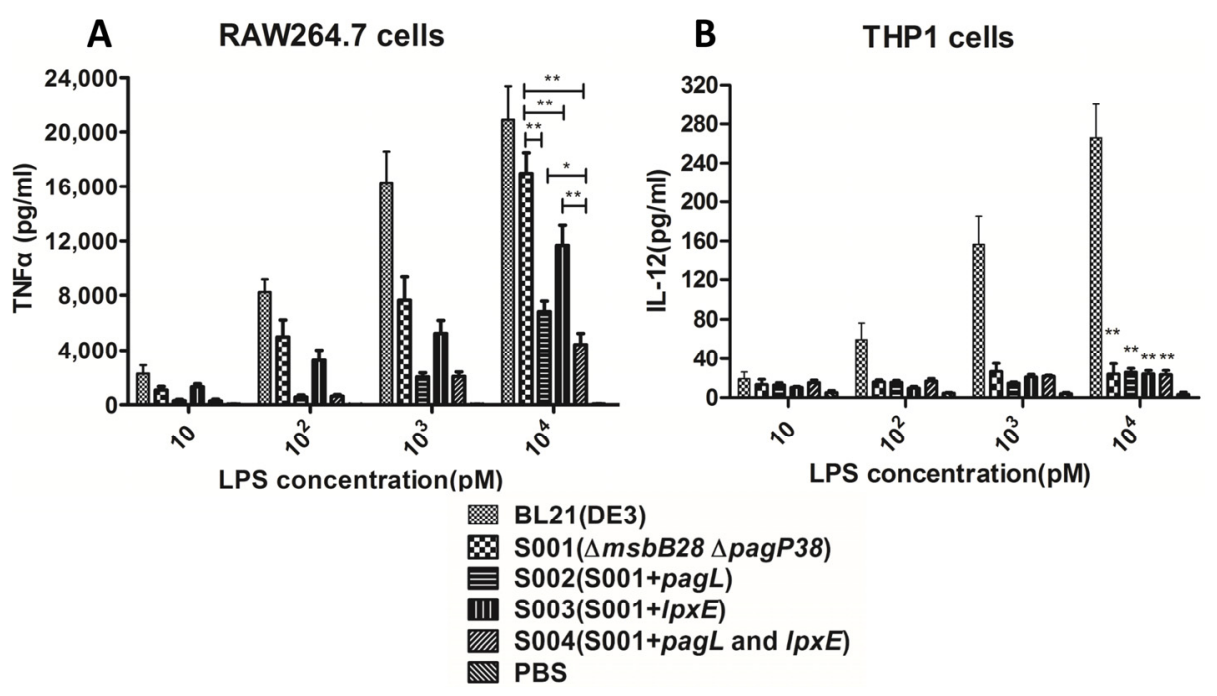

Figure 5. Cytokine concentrations in culture supernatants of RAW264.7 cells and THP1 cells stimulated by different concentrations of lipopolysaccharide (LPS) samples from E. coli BL21 (DE3) and mutant strains. Supernatant samples from cells were collected after $24 \mathrm{~h}$ stimulation with LPS and assayed for the production of inflammatory factor. (A) TNF- $\alpha$ released into the culture supernatant of RAW264.7 cells stimulated for $24 \mathrm{~h}$ with different concentration of LPS; (B) IL-12 released into the culture supernatant of THP1 cells stimulated for $24 \mathrm{~h}$ with different concentration of LPS. One-way analysis of variance was used to evaluate differences in cytokine concentrations, significant differences between the parental strain groups and other mutant strain groups stimulated by LPS were shown. $* p<0.05 ; * * p<0.01$. The experiment was performed in triplicate, and data are shown as means \pm standard deviation, with PBS group as negative control.

At all concentrations, the level of TNF- $\alpha$ in the RAW264.7 stimulated by LPS from BL21 (DE3) was significantly higher than that stimulated by other modified LPS, indicating that hexa-acylated lipid A from the wild-type $E$. coli strain is the most endotoxic form of these strains in activating the TLR4-pathway, with the TNF- $\alpha$ levels from $2300 \mathrm{pg} / \mathrm{mL}$ to $20,900 \mathrm{pg} / \mathrm{mL}$ induced by BL21 (DE3) LPS at $10 \mathrm{pmol} / \mathrm{L}$ to $10^{4} \mathrm{pmol} / \mathrm{L}$. The LPS purified from S001 ( $\triangle m s b B 28 \Delta p a g P 38$ ) induced significantly higher levels of TNF- $\alpha$ than did other S001 expressing pagL, or lpxE or both, and LPS purified from S004 (pagL and lpxE) induced the significantly lower levels of the pro-inflammatory cytokines TNF- $\alpha$ than did the S002 (pagL) and S003 (lpxE), but still induced $4427 \mathrm{pg} / \mathrm{mL}$ TNF- $\alpha$ in the mouse 
macrophage cell line RAW264.7 at $10^{4} \mathrm{pmol} / \mathrm{L}$, indicating that monophosphoryl tetra-acylated lipid A still acts as an agonist when interacting with mouse TLR4.

The levels of IL-12 released by human THP1 cells were proportional to the concentration of LPS isolated from the parent strain BL21 (DE3) and its derivatives. The IL-12 levels from $19.4 \mathrm{pg} / \mathrm{mL}$ to $266 \mathrm{pg} / \mathrm{mL}$ were induced by BL21 (DE3) LPS at $10 \mathrm{pmol} / \mathrm{L}$ to $10^{4} \mathrm{pmol} / \mathrm{L}$. The LPS from other BL21 (DE3) derivatives showed the ability to stimulate lower levels of the production of IL-12 in the THP1 cell line, but the levels of IL-12 did not increase proportionally to each LPS concentration. No difference of the levels of IL-12 in the THP1 cell line was observed when stimulation of the LPS from the S001 ( $\triangle m s b B 28 \Delta p a g P 38)$ and the S001 expressing pagL, or lpxE or both, indicating that penta-acylated lipid A from S001 ( $\triangle m s b B 28 \Delta p a g P 38)$ containing five fatty acyl chain and further modified lipid A behaves as an antagonist that does not activate human TLR4 receptor [16,17].

\subsection{Lipid A Modification Did Not Change the Growth Kinetics of the BL21 and Its Derivatives and They Retain Ability to Produce Recombinant Proteins}

We measured the growth curves of the BL21 (DE3) and its derivatives to investigate whether the lipid A modification influences the bacterial growth as it is an essential feature to be considered if the bacterial strains would be used as hosts to express heterologous proteins. The modification of lipid A had no significant effect on growth rates of the BL21 (DE3) derivatives. The growth rates of all the derivative strains were similar to that of the parent strain BL21 (DE3) (Figure 6A); therefore, these BL21 derivative strains with low entodoxic lipid A were suitable for application of expressing recombinant proteins.

A

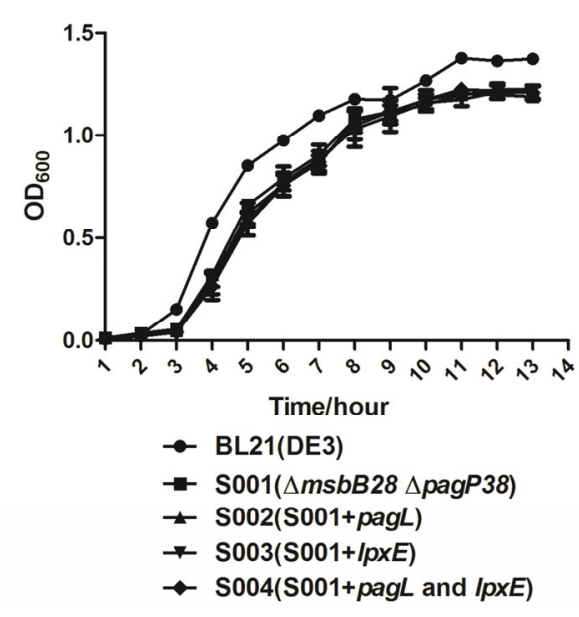

B

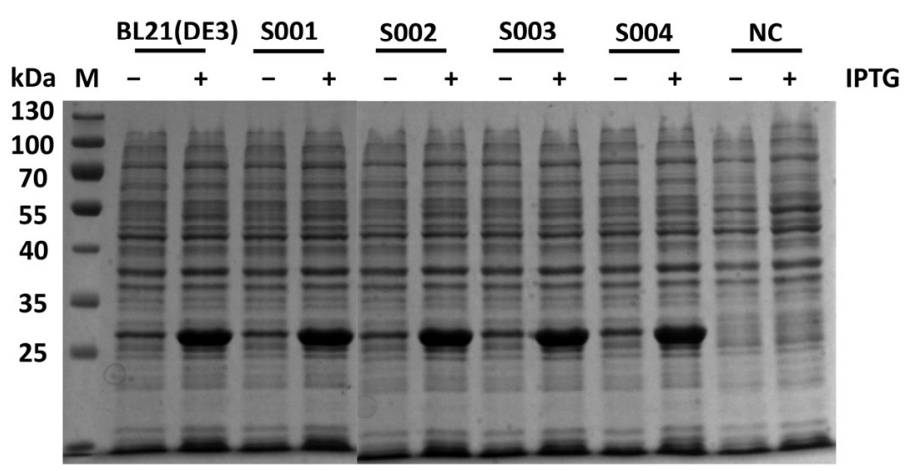

Figure 6. Biological characteristics analysis of BL21 (DE3) mutant strains. (A) Comparison of growth of BL21 (DE3) mutants, the experiment was performed in triplicate, and data were shown as means \pm standard deviation; (B) Expression of PhoP in BL21 (DE3) and mutant strains. The recombinant proteins and strain used for expression are indicated in this figure. M: Protein ladder; 1: BL21 (DE3); 2: S001 ( $\triangle m s b B 28$ ApagP38); 3: S002 $(\mathrm{S} 001+$ pagL $) ; 4$ : S003 (S001 + lpxE); 5: S004 (S001 + pagL and lpxE); NC: Negative control, BL21 (DE3) containing the control plasmid. 
We then investigated their abilities to produce recombinant proteins in the BL21 (DE3) and its derivatives (Figure 6B). The comparison was performed using the protein PhoP, a global regulatory protein from Pasteurella multocida 8081, for their variable levels of expression in BL21 (DE3). No significant differences were observed between the four mutant strains and the parent BL21 strain in the levels of recombinant protein production after induction by IPTG, indicating that the lipid A modification did not compromise the ability to produce the recombinant protein.

\section{Discussion}

BL21 (DE3) and its derivatives are widely used for protein expression and purification for many purposes. One concern when using these proteins for clinical purposes is LPS contamination, leading to unacceptable reactogenicity. Therefore, the endotoxic LPS has to be removed from recombinant proteins before their use for therapeutic purpose. Previous studies demonstrated that a non-myristoylated (penta-acylated) LPS resulting from inactivation of $m s b B$ in E. coli and Salmonella exhibited markedly less ability to induce pro-inflammatory TNF- $\alpha$ in human monocytes $[14,39,49]$. To minimize the endotoxic activity of the LPS, lipid A structure was further modified in this study by removal of 3-O-acylation chain and phosphate group in the BL21 (DE3) $m s b B$ mutant using plasmids to express pagL and lpxE genes. We also included pagP deletion in the BL21 (DE3) to avoid the potential addition of palmitate chain to the lipid A [20,50]. Initially, we planned to inactivate $h t r B$ gene in the $m s b B$ mutant, which would result in production of tetra-acylated lipid A, but the double mutant exhibited abnormal growth in the LB media (data not shown). We then opted to select the pagL to selectively remove 3-O-acylation of lipid A [51,52].

The MS data demonstrated that PagL and LpxE expression from plasmid could lead to complete production of tetra-acylated lipid A and mono-phosphorylated penta-acylated lipid A, and both PagL and LpxE expression in one plasmid resulted in production of mono-phosphorylated tetra-acylated lipid A while minor decorations such as pEtN or Ara4N were present in the lipid A (Figures 2 and 3) [27]. No data were currently available to demonstrate whether these minor modifications on the phosphate position of lipid A impact its interaction with the TLR4 receptor. Therefore, in this study we still retained arnT and eptA genes, which are responsible for $\mathrm{pEtN}$ or Ara4N addition to the lipid A, in the BL21 (DE3) chromosome [53,54].

Toll-like receptors (TLRs) have an essential function in innate and adaptive immunity by responding to microbial components, and they are expressed mainly on antigen-presenting cells such as monocytes-macrophages and dendritic cells [7]. TLR4, as the receptor for the Gram-negative bacterial LPS, can mediate the signal of macrophages for cytokine production such as IL-6, IL12 and TNF- $\alpha[15,16,28]$. We therefore chose the macrophage cell line RAW264.7, originated from mouse, and THP1 cells derived from human to investigate the effect of LPS modification to TLR4 activity and evaluate the endotoxic activity of that lipid A in mammalian and human cells.

Previous reports show $m s b B$ deletions in BL21 (DE3) and that other bacteria reduce the capacity of LPS from these $m s b B$ mutants to activate human-derived immature monocyte-derived DC by more than two orders of magnitude [16,55]. Similarly, we have shown that LPS isolated from S001 ( $\triangle m s b B 28 \triangle p a g P 38$ ) also has strongly reduced its capacity to stimulate IL12 production by human THP1, but still retained its capacity to trigger TNF- $\alpha$ in RAW264.7 cells derived from mouse (Figure 5). 
Removal of the 3-O-acylation chain or phosphate group in the penta-acylated LPS produced by S001 $(\triangle m s b B 28 \Delta p a g P 38)$ resulted in greatly decreased capacity to trigger TNF-a production in RAW264.7, and the former significantly reduced the potency in triggering TNF- $\alpha$ production in RAW264.7 compared with effect of removal of phosphate group (Figure 5), indicating that alteration of fatty acid number of lipid A would be a more efficient approach to decrease its endotoxic activity than removal of phosphate group [27,56]. While LPS isolated from the S003 ( $p a g L$ and $\operatorname{lpxE}$ ) greatly reduced its ability to induce TNF- $\alpha$ in the RAW264.7 cell, this mono-phosphorated tetra-acylated LPS induces pro-inflammatory response in the mouse cell. Further modifications of the lipid A of the BL21 (DE3) to minimize its endotoxic activity by other approaches, such as inactivation of $h \operatorname{tr} B$ and removal of the second phosphate group by $l p x F$, can remove the 4 '-phosphate group $[57,58]$.

BL21 (DE3) and its derivatives were tested for expression of recombinant proteins from pET32a plasmids encoding by phoP and other genes from Pasteurella multocida 8081 and Salmonella typhimurium. While lipid A modification has minor effects on bacterial growth, the levels of expression were indistinguishable from the original BL21 (DE3) strain (Figure 6 and data not shown), which was consistent with the previous reports [16]. This also provided us with a basis to genetically engineer the BL21 (DE3) to further reduce its endotoxic activity for a future study.

We chose plasmid vectors to express $p a g L$ and $\operatorname{lpxE}$ because it is convenient for transferring plasmids to other bacterial strains for other purposes; for example, plasmid pQK004 carrying lpxE or pQK005 carrying $\operatorname{lp} x E$ and $p a g L$ can be used for production of mono-phosphoryl lipid A, which can be used as vaccine adjuvants [26,27]. While insertion of these genes into the bacterial chromosome could avoid utilization of selection antibiotics, these chromosome-integrated genes are not always expressed enough for fully modifying the lipid A structure [27,37]; moreover, we can take advantage of balanced-lethal host-vector systems to stably maintain plasmids without use of antibiotic as selective pressures in BL21 (DE3) or other bacterial strains [59,60]. Therefore, plasmid vector is still an ideal option to express heterologous genes in E. coli.

\section{Conclusions}

In all, in this study we have succeeded in constructing a BL21 $\Delta m s b B$ mutant and its derivatives, and demonstrated that these progeny strains contained modified forms of lipid A with markedly decreased capacity to induce the inflammatory response in human and mouse cells. These mutant strains may be used for expressing therapeutic recombinant proteins or targeted drugs without elimination of LPS.

\section{Acknowledgments}

We thank Rey C. Allen from the Biodesign Institute at Arizona State University for editing this manuscript. This study was supported by the National Natural Science Foundation of China (31270981, 31200697). 


\section{Author Contributions}

Qiong Liu, Qing Liu and Qingke Kong conceived and designed the experiments; Qiong Liu, Yanyan Li, Xinxin Zhao performed the experiments; Qiong Liu, Qing Liu and Qingke Kong analyzed the data; Xue Yang coordinated this project; Qiong Liu and Qingke Kong wrote this paper.

\section{Conflict of Interest}

The authors declare no conflict of interest.

\section{References}

1. Kong, Q.; Yang, J.; Liu, Q.; Alamuri, P.; Roland, K.L.; Curtiss, R. Effect of deletion of genes involved in lipopolysaccharide core and $\mathrm{O}$-antigen synthesis on virulence and immunogenicity of Salmonella enterica serovar Typhimurium. Infect. Immun. 2011, 79, 4227-4239.

2. Shiloach, J.; Kaufman, J.; Guillard, A.; Fass, R. Effect of glucose supply strategy on acetate accumulation, growth, and recombinant protein production by Escherichia coli BL21 ( $\lambda \mathrm{DE} 3)$ and Escherichia coli JM109. Biotechnol. Bioeng. 1996, 49, 421-428.

3. Colucci, M.; Balconi, G.; Lorenzet, R.; Pietra, A.; Locati, D.; Donati, M.; Semeraro, N. Cultured human endothelial cells generate tissue factor in response to endotoxin. J. Clin. Investig. 1983, 71, 1893-1896.

4. Ziegler, E.J.; Fisher, C.J., Jr.; Sprung, C.L.; Straube, R.C.; Sadoff, J.C.; Foulke, G.E.; Wortel, C.H.; Fink, M.P.; Dellinger, R.P.; Teng, N.N. Treatment of Gram-negative bacteremia and septic shock with HA-1A human monoclonal antibody against endotoxin: A randomized, double-blind, placebo-controlled trial. N. Engl. J. Med. 1991, 324, 429-436.

5. Petsch, D.; Anspach, F.B. Endotoxin removal from protein solutions. J. Biotechnol. 2000, 76, 97-119.

6. Galanos, C.; Lüderitz, O.; Rietschel, E.T.; Westphal, O.; Brade, H.; Brade, L.; Freudenberg, M.; Schade, U.; Imoto, M.; Yoshimura, H. Synthetic and natural Escherichia coli free lipid A express identical endotoxic activities. Eur. J. Biochem. 1985, 148, 1-5.

7. Raetz, C.R.; Whitfield, C. Lipopolysaccharide endotoxins. Annu. Rev. Biochem. 2002, 71, 635-700.

8. Needham, B.D.; Carroll, S.M.; Giles, D.K.; Georgiou, G.; Whiteley, M.; Trent, M.S. Modulating the innate immune response by combinatorial engineering of endotoxin. Proc. Natl. Acad. Sci. USA 2013, 110, 1464-1469.

9. Piya, M.K.; McTernan, P.G.; Kumar, S. Adipokine inflammation and insulin resistance: The role of glucose, lipids and endotoxin. J. Endocrinol. 2013, 216, doi:10.1530/JOE-12-0498.

10. Xia, D.; Samols, D. Transgenic mice expressing rabbit C-reactive protein are resistant to endotoxemia. Proc. Natl. Acad. Sci. USA 1997, 94, 2575-2580.

11. Raetz, C.R.; Guan, Z.; Ingram, B.O.; Six, D.A.; Song, F.; Wang, X.; Zhao, J. Discovery of new biosynthetic pathways: The lipid A story. J. Lipid Res. 2009, 50, doi:10.1194/jlr.R800060-JLR200. 
12. Trent, M.S.; Pabich, W.; Raetz, C.R.; Miller, S.I. A PhoP/PhoQ-induced lipase (PagL) that catalyzes 3-O-deacylation of lipid A precursors in membranes of Salmonella typhimurium. J. Biol. Chem. 2001, 276, 9083-9092.

13. Somerville, J.E.; Cassiano, L.; Darveau, R.P. Escherichia coli $m s b B$ gene as a virulence factor and a therapeutic target. Infect. Immun. 1999, 67, 6583-6590.

14. Somerville, J.E., Jr.; Cassiano, L.; Bainbridge, B.; Cunningham, M.D.; Darveau, R.P. A novel Escherichia coli lipid A mutant that produces an antiinflammatory lipopolysaccharide. J. Clin. Investig. 1996, 97, 359-365.

15. Coats, S.R.; Pham, T.-T.T.; Bainbridge, B.W.; Reife, R.A.; Darveau, R.P. MD-2 mediates the ability of tetra-acylated and penta-acylated lipopolysaccharides to antagonize Escherichia coli lipopolysaccharide at the TLR4 signaling complex. J. Immunol. 2005, 175, 4490-4498.

16. Cognet, I.; de Coignac, A.B.; Magistrelli, G.; Jeannin, P.; Aubry, J.-P.; Maisnier-Patin, K.; Caron, G.; Chevalier, S.; Humbert, F.; Nguyen, T. Expression of recombinant proteins in a lipid A mutant of Escherichia coli BL21 with a strongly reduced capacity to induce dendritic cell activation and maturation. J. Immunol. Methods 2003, 272, 199-210.

17. Low, K.B.; Ittensohn, M.; Le, T.; Platt, J.; Sodi, S.; Amoss, M.; Carmichael, E.; Chakraborty, A.; Ash, O.; Fischer, J. Lipid A mutant Salmonella with suppressed virulence and TNFa induction retain tumor-targeting in vivo. Nat. Biotechnol. 1999, 17, 37-41.

18. Bishop, R.E.; Gibbons, H.S.; Guina, T.; Trent, M.S.; Miller, S.I.; Raetz, C.R. Transfer of palmitate from phospholipids to lipid A in outer membranes of Gram-negative bacteria. EMBO J. 2000, 19, 5071-5080.

19. Guo, L.; Lim, K.B.; Poduje, C.M.; Daniel, M.; Gunn, J.S.; Hackett, M.; Miller, S.I. Lipid A acylation and bacterial resistance against vertebrate antimicrobial peptides. Cell 1998, 95, 189-198.

20. Bishop, R.E. The lipid A palmitoyltransferase PagP: Molecular mechanisms and role in bacterial pathogenesis. Mol. Microbiol. 2005, 57, 900-912.

21. Hwang, P.M.; Choy, W.-Y.; Lo, E.I.; Chen, L.; Forman-Kay, J.D.; Raetz, C.R.; Privé, G.G.; Bishop, R.E.; Kay, L.E. Solution structure and dynamics of the outer membrane enzyme PagP by NMR. Proc. Natl. Acad. Sci. USA 2002, 99, 13560-13565.

22. Brown, D.B.; Muszyński, A.; Salas, O.; Speed, K.; Carlson, R.W. Elucidation of the 3-O-deacylase gene, pagL, required for the removal of primary $\beta$-hydroxy fatty acid from the lipid $\mathrm{A}$ in the nitrogen-fixing endosymbiont Rhizobium etli CE3. J. Biol. Chem. 2013, 288, 12004-12013.

23. Kawasaki, K.; Teramoto, M.; Tatsui, R.; Amamoto, S. Lipid A 3'-O-deacylation by Salmonella outer membrane enzyme LpxR modulates the ability of lipid A to stimulate Toll-like receptor 4. Biochem. Biophys. Res. Commun. 2012, 428, 343-347.

24. Geurtsen, J.; Steeghs, L.; Hamstra, H.-J.; ten Hove, J.; de Haan, A.; Kuipers, B.; Tommassen, J.; van der Ley, P. Expression of the lipopolysaccharide-modifying enzymes PagP and PagL modulates the endotoxic activity of Bordetella pertussis. Infect. Immun. 2006, 74, 5574-5585.

25. Geurtsen, J.; Steeghs, L.; ten Hove, J.; van der Ley, P.; Tommassen, J. Dissemination of lipid A deacylases (PagL) among Gram-negative bacteria identification of active-site histidine and serine residues. J. Biol. Chem. 2005, 280, 8248-8259. 
26. Wang, X.; Karbarz, M.J.; McGrath, S.C.; Cotter, R.J.; Raetz, C.R. MsbA Transporter-dependent lipid A 1-Dephosphorylation on the periplasmic surface of the inner membrane topography of Francisella novicida LpxE expressed in Escherichia coli. J. Biol. Chem. 2004, 279, 49470-49478.

27. Kong, Q.; Six, D.A.; Roland, K.L.; Liu, Q.; Gu, L.; Reynolds, C.M.; Wang, X.; Raetz, C.R.; Curtiss, R. Salmonella synthesizing 1-monophosphorylated lipopolysaccharide exhibits low endotoxic activity while retaining its immunogenicity. J. Immunol. 2011, 187, 412-423.

28. Kayagaki, N.; Wong, M.T.; Stowe, I.B.; Ramani, S.R.; Gonzalez, L.C.; Akashi-Takamura, S.; Miyake, K.; Zhang, J.; Lee, W.P.; Muszyński, A. Noncanonical inflammasome activation by intracellular LPS independent of TLR4. Science 2013, 341, 1246-1249.

29. Park, B.S.; Song, D.H.; Kim, H.M.; Choi, B.-S.; Lee, H.; Lee, J.-O. The structural basis of lipopolysaccharide recognition by the TLR4-MD-2 complex. Nature 2009, 458, 1191-1195.

30. Rietschel, E.T.; Kirikae, T.; Schade, F.U.; Mamat, U.; Schmidt, G.; Loppnow, H.; Ulmer, A.J.; Zähringer, U.; Seydel, U.; di Padova, F. Bacterial endotoxin: Molecular relationships of structure to activity and function. FASEB J. 1994, 8, 217-225.

31. Rietschel, E.T.; Kirikae, T.; Schade, F.U.; Ulmer, A.J.; Holst, O.; Brade, H.; Schmidt, G.; Mamat, U.; Grimmecke, H.-D.; Kusumoto, S. The chemical structure of bacterial endotoxin in relation to bioactivity. Immunobiology 1993, 187, 169-190.

32. Brandenburg, K.; Wiese, A. Endotoxins: Relationships between structure, function, and activity. Curr. Top. Med. Chem. 2004, 4, 1127-1146.

33. Miroux, B.; Walker, J.E. Over-production of proteins in Escherichia coli: Muztant hosts that allow synthesis of some membrane proteins and globular proteins at high levels. J. Mol. Biol. 1996, 260, 289-298.

34. Nakayama, K.; Kelly, S.M.; Curtiss, R. Construction of an $\mathrm{ASD}^{+}$expression-cloning vector: Stable maintenance and high level expression of cloned genes in a Salmonella vaccine strain. Nat. Biotechnol. 1988, 6, 693-697.

35. Sambrook, J.; Russell, D.W.; Russell, D.W. Molecular Cloning: A Laboratory Manual (3-Volume Set); Cold Spring Harbor Laboratory Press: Cold Spring Harbor, NY, USA, 2001.

36. Edwards, R.A.; Keller, L.H.; Schifferli, D.M. Improved allelic exchange vectors and their use to analyze 987P fimbria gene expression. Gene 1998, 207, 149-157.

37. Roland, K.; Curtiss, R., III; Sizemore, D. Construction and evaluation of a $\Delta$ cya $\Delta$ crp Salmonella typhimurium strain expressing avian pathogenic Escherichia coli O78 LPS as a vaccine to prevent airsacculitis in chickens. Avian Dis. 1999, 43, 429-441.

38. Zhang, X.; Kong, W.; Ashraf, S.; Curtiss, R. A one-plasmid system to generate influenza virus in cultured chicken cells for potential use in influenza vaccine. J. Virol. 2009, 83, 9296-9303.

39. Kong, Q.; Six, D.A.; Liu, Q.; Gu, L.; Roland, K.L.; Raetz, C.R.; Curtiss, R., III. Palmitoylation state impacts induction of innate and acquired immunity by the Salmonella enterica serovar Typhimurium msbB mutant. Infect. Immun. 2012, 80, 1302.

40. Kang, H.Y.; Dozois, C.M.; Tinge, S.A.; Lee, T.H.; Curtiss, R., III. Transduction-mediated transfer of unmarked deletion and point mutations through use of counterselectable suicide vectors. J. Bacteriol. 2002, 184, 307-312. 
41. Li, Y.; Powell, D.A.; Shaffer, S.A.; Rasko, D.A.; Pelletier, M.R.; Leszyk, J.D.; Scott, A.J.; Masoudi, A.; Goodlett, D.R.; Wang, X. LPS remodeling is an evolved survival strategy for bacteria. Proc. Natl. Acad. Sci. USA 2012, 109, 8716-8721.

42. Apicella, M.A.; Griffiss, J.; Schneider, H. Isolation and characterization of lipopolysaccharides, lipooligosaccharides, and lipid A. Methods Enzymol. 1994, 235, 242-252.

43. Osborn, M.J. Studies on the Gram-negative cell wall, I. Evidence for the role of 2-keto-3-deoxyoctonate in the lipopolysaccharide of Salmonella typhimurium. Proc. Natl. Acad. Sci. USA 1963, 50, 499-506.

44. Bligh, E.G.; Dyer, W.J. A rapid method of total lipid extraction and purification. Can. J. Biochem. Physiol. 1959, 37, 911-917.

45. Trent, M.S.; Ribeiro, A.A.; Lin, S.; Cotter, R.J.; Raetz, C.R. An inner membrane enzyme in Salmonella and Escherichia coli that transfers 4-amino-4-deoxy-1-arabinose to lipid A induction in polymyxin-resistant mutants and role of novel lipid-linked donor. J. Biol. Chem. 2001, 276, $43122-43131$.

46. Sims, K.; Haynes, C.A.; Kelly, S.; Allegood, J.C.; Wang, E.; Momin, A.; Leipelt, M.; Reichart, D.; Glass, C.K.; Sullards, M.C. Kdo2-lipid A, a TLR4-specific agonist, induces de novo sphingolipid biosynthesis in RAW264.7 macrophages, which is essential for induction of autophagy. J. Biol. Chem. 2010, 285, 38568-38579.

47. Bès-Houtmann, S.; Roche, R.; Hoareau, L.; Gonthier, M.-P.; Festy, F.; Caillens, H.; Gasque, P.; d'Hellencourt, C.L.; Cesari, M. Presence of functional TLR2 and TLR4 on human adipocytes. Histochem. Cell Biol. 2007, 127, 131-137.

48. Hirschfeld, M.; Ma, Y.; Weis, J.H.; Vogel, S.N.; Weis, J.J. Cutting edge: Repurification of lipopolysaccharide eliminates signaling through both human and murine toll-like receptor 2. J. Immunol. 2000, 165, 618-622.

49. Khan, S.A.; Everest, P.; Servos, S.; Foxwell, N.; Zähringer, U.; Brade, H.; Rietschel, E.T.; Dougan, G.; Charles, I.G.; Maskell, D.J. A lethal role for lipid A in Salmonella infections. Mol. Microbiol. 1998, 29, 571-579.

50. Jia, W.; el Zoeiby, A.; Petruzziello, T.N.; Jayabalasingham, B.; Seyedirashti, S.; Bishop, R.E. Lipid trafficking controls endotoxin acylation in outer membranes of Escherichia coli. J. Biol. Chem. 2004, 279, 44966-44975.

51. Johnson, D.A.; Keegan, D.S.; Sowell, C.G.; Livesay, M.T.; Johnson, C.L.; Taubner, L.M.; Harris, A.; Myers, K.R.; Thompson, J.D.; Gustafson, G.L. 3-O-Desacyl monophosphoryl lipid A derivatives: Synthesis and immunostimulant activities. J. Med. Chem. 1999, 42, 4640-4649.

52. Imoto, M.; Yoshimura, H.; Shimamoto, T.; Sakaguchi, N.; Kusumoto, S.; Shiba, T. Total synthesis of Escherichia coli lipid A, the endotoxically active principle of cell-surface lipopolysaccharide. Bull. Chem. Soc. Jpn. 1987, 60, 2205-2214.

53. Tran, A.X.; Lester, M.E.; Stead, C.M.; Raetz, C.R.; Maskell, D.J.; McGrath, S.C.; Cotter, R.J.; Trent, M.S. Resistance to the antimicrobial peptide polymyxin requires myristoylation of Escherichia coli and Salmonella typhimurium lipid A. J. Biol. Chem. 2005, 280, 28186-28194.

54. Doerrler, W.T.; Gibbons, H.S.; Raetz, C.R. MsbA-dependent translocation of lipids across the inner membrane of Escherichia coli. J. Biol. Chem. 2004, 279, 45102-45109. 
55. Van Der Ley, P.; Steeghs, L.; Hamstra, H.J.; ten Hove, J.; Zomer, B.; van Alphen, L. Modification of lipid A biosynthesis in Neisseria meningitidis 1pxL mutants: Influence on lipopolysaccharide structure, toxicity, and adjuvant activity. Infect. Immun. 2001, 69, 5981-5990.

56. Morrison, D.C.; Silverstein, R.; Luchi, M.; Shnyra, A. Structure-function relationships of bacterial endotoxins: Contribution to microbial sepsis. Infect. Dis. Clin. N. Am. 1999, 13, 313-340.

57. Kong, Q.; Six, D.A.; Liu, Q.; Gu, L.; Wang, S.; Alamuri, P.; Raetz, C.R.; Curtiss, R. Phosphate groups of lipid A are essential for Salmonella enterica serovar Typhimurium virulence and affect innate and adaptive immunity. Infect. Immun. 2012, 80, 3215-3224.

58. Karow, M.; Georgopoulos, C. Isolation and characterization of the Escherichia coli msbB gene, a multicopy suppressor of null mutations in the high-temperature requirement gene $h$ tr $B$. J. Bacteriol. 1992, 174, 702-710.

59. Kim, K.; Jeong, J.H.; Lim, D.; Hong, Y.; Yun, M.; Min, J.-J.; Kwak, S.-J.; Choy, H.E. A novel balanced-lethal host-vector system based on glmS. PLOS ONE 2013, 8, e60511.

60. Galán, J.E.; Nakayama, K.; Curtiss, R. Cloning and characterization of the asd gene of Salmonella typhimurium: Use in stable maintenance of recombinant plasmids in Salmonella vaccine strains. Gene 1990, 94, 29-35.

(C) 2015 by the authors; licensee MDPI, Basel, Switzerland. This article is an open access article distributed under the terms and conditions of the Creative Commons Attribution license (http://creativecommons.org/licenses/by/4.0/). 